\title{
An Analytical Study on Bistability of Fabry-Perot Semiconductor Optical Amplifiers
}

\author{
Gang $\mathrm{WANG}^{*}$, Shuqiang CHEN, and Huajun YANG \\ School of Physical Electronics, University of Electronic Science and Technology of China, Chengdu, 610054, China \\ *Corresponding author: Gang WANG_E-mail: buncan_wang@126.com
}

\begin{abstract}
Optical bistabilities have been considered to be useful for sensor applications. As a typical nonlinear device, Fabry-Perot semiconductor optical amplifiers (FPSOAs) exhibit bistability under certain conditions. In this paper, the bistable characteristics in FPSOAs are investigated theoretically. Based on Adams's relationship between the incident optical intensity $I_{\text {in }}$ and the $z$-independent average intracavity intensity $I_{\mathrm{av}}$, an analytical expression of the bistable loop width in SOAs is derived. Numerical simulations confirm the accuracy of the analytical result.
\end{abstract}

Keywords: Bistability; Fabry-Perot resonator; semiconductor optical amplifiers

Citation: Gang WANG, Shuqiang CHEN, and Huajun YANG, "An Analytical Study on Bistability of Fabry-Perot Semiconductor Optical Amplifiers," Photonic Sensors, 2016, 6(3): 268-273.

\section{Introduction}

Optical bistability (OB) has been found to be a beneficial technology for designing optical sensors [1-2]. It is well known that Fabry-Perot semiconductor optical amplifiers (FPSOAs) can operate in a bistable regime with proper parameters. Therefore, further studies on bistability of FPSOAs are worthwhile from a practical point of view. In [3], the authors presented a commonly-used model for investigating the bistability in SOAs. By use of this model, the static and dynamic bistable characteristics in different types of SOAs have been investigated substantially [3-8]. However, these studies have been performed either numerically or experimentally. So far, few analytical results on the bistability in SOAs have been presented. In this paper, based on Adams's model [3], the necessary condition for bistability in SOAs is deduced. Moreover, analytical expressions of the switch-up power, switch-down power of the incident beam, and the width of hysteresis loop in SOAs are derived. Numerical calculations are carried out to verify the analytical results.

The remainder of this paper is organized as follows. Section 2 gives detailed derivations of the necessary condition for bistability and the expression of hysteresis loop width in an FPSOA. Section 3 affords the numerical experiments to verify the correctness of the analytical results. Discussion and conclusions are provided in Section 4.

\section{Theoretical analysis}

Considering an SOA, it is assumed that in the cavity the optical intensity is uniform and the spontaneous emission is neglected. In the following, the analysis is carried out according to the relationships derived by Adams [3], in which the input intensity $I_{\text {in }}$, the output intensity $I_{\text {out }}$, and the

Received: 17 April 2016 / Revised: 2 June 2016

(C) The Author(s) 2016. This article is published with open access at Springerlink.com DOI: $10.1007 / \mathrm{s} 13320-016-0332-\mathrm{x}$

Article type: Regular 
$z$-independent intracavity intensity $I_{\mathrm{av}}$ could be expressed as follows.

$$
\begin{gathered}
\frac{I_{\text {out }}}{I_{s}}=\frac{I_{\text {in }}}{I_{s}} \cdot \frac{\left(1-R_{1}\right)\left(1-R_{2}\right) e^{g L}}{\left(1-\sqrt{R_{1} R_{2}} e^{g L}\right)^{2}+4 \sqrt{R_{1} R_{2}} e^{g L} \sin ^{2} \phi} \\
\frac{I_{\text {av }}}{I_{s}}=\frac{I_{\text {out }}}{I_{s}} \cdot \frac{\left(1-e^{-g L}\right)\left(1+R_{2} e^{g L}\right)}{\left(1-R_{2}\right) g L}
\end{gathered}
$$

where $R_{1}$ and $R_{2}$ are the reflectivities of the two cavity mirrors, $g$ is the net gain per unit length, $\phi$ is the single-pass phase change, and $L$ is the cavity length. In the steady state, $g$ and $\phi$ can be expressed as

$$
\begin{aligned}
& g=\frac{\Gamma g_{0}}{1+I_{\mathrm{av}} / I_{s}}-\alpha \\
& \phi=\phi_{0}+\frac{g_{0} L b}{2}\left(\frac{I_{\mathrm{av}} / I_{s}}{1+I_{\mathrm{av}} / I_{s}}\right) \\
& g_{0}=a\left(n-n_{0}\right)
\end{aligned}
$$

where $\Gamma$ is the optical confinement factor, $\alpha$ is the effective loss coefficient, $g_{0}$ is the unsaturated material gain, $\phi_{0}$ is the initial phase detuning, $I_{\mathrm{s}}$ is the saturation optical intensity, $b$ is the linewidth enhancement factor, $n$ is the carrier density, $a$ is the gain coefficient, and $n_{0}$ is the carrier density at transparency.

From (1) to (5), the input-output (IO) hysteresis loop can be observed by numerical simulation under certain parameter values [3]. In this paper, we analytically calculate the bistability in SOAs. From the numerical results and the definition of the bistability, it is obvious that in the bistable operation region, for a given input intensity $I_{\text {in }}$, there are three corresponding solutions of the output intensity $I_{\text {out }}$ to (1)-(5). Namely, one is the unstable-state solution, and the other two are the stable-state solutions. Equation (2) shows that $I_{\text {out }}$ is a single valued function of $I_{\mathrm{av}}$. Thereby, it can be inferred that in the region where the hysteresis loop appears, there exist three values of $I_{\text {av }}$ corresponding to one value of $I_{\text {in }}$. To ensure this feature, the function $I_{\mathrm{in}}\left(I_{\mathrm{av}}\right)$ should have two extreme values in this region. Hence, from the mathematical point of view, the necessary condition for bistability of SOA is that the first-order derivative of the function $I_{\text {in }}\left(I_{\text {av }}\right)$ should have two zero points.

From (1) and (2), one can obtain the relationship between $I_{\text {in }}$ and $I_{\text {av }}$ :

$$
\frac{I_{\text {in }}}{I_{s}}=\frac{\left(1-\sqrt{R_{1} R_{2}} e^{g L}\right)^{2}+4 \sqrt{R_{1} R_{2}} e^{g L} \sin ^{2} \phi}{\left(1-R_{1}\right)\left(e^{g L}-1\right)\left(1+R_{2} e^{g L}\right)} \cdot g L \cdot \frac{I_{\mathrm{av}}}{I_{s}} .
$$

For convenience, let $x=I_{\mathrm{av}} / I_{s}, y=I_{\mathrm{in}} / I_{s}$. Then (6) can be rewritten as

$$
y=\frac{\ln (G) \cdot\left[\left(1-\sqrt{R_{1} R_{2}} G\right)^{2}+4 \sqrt{R_{1} R_{2}} G \sin ^{2} \phi\right]}{\left(1-R_{1}\right)(G-1)\left(1+R_{2} G\right)} \cdot x
$$

where $G=\exp (g L)$ represents the single pass gain in the cavity.

Clearly, (7) owns a complicated form, which makes it difficult to obtain the zero point of the first-order derivative of $y$. In order to theoretically find the necessary condition for the occurrence of hysteresis loop of SOAs and further achieve analytical results on the bistability, some approximations are demanded to simplify the form of (7). Naturally, the right hand of (7) can be expanded into Taylor series in terms of $x$. Since we try to examine whether the first-order derivative of $y$ has two zero points, it is reasonable that three-order approximation of the Taylor series of $y$ is adopted. Thus $y$ can be rewritten as follows:

$$
\begin{aligned}
y \approx & F_{0}+F_{1} \cdot\left(x-x_{0}\right)+\frac{1}{2 !} F_{2} \cdot\left(x-x_{0}\right)^{2}+ \\
& \frac{1}{3 !} F_{3} \cdot\left(x-x_{0}\right)^{3}
\end{aligned}
$$

where $F_{0}=y\left(x_{0}\right)$ and $F_{i}=\left.\frac{d^{i} y}{d x^{i}}\right|_{x=x_{0}}(1 \leq i \leq 3)$ are the $i$ th order derivatives of $y$ at the reference point $x=x_{0}$. In the following, (8) is a start point for further deduction and analysis. After straight and complex computation, the coefficients in (8) can be derived in the following form:

$$
\begin{aligned}
\frac{d y}{d x}= & \frac{d T_{1}}{d x} x+T_{1}+\frac{d T_{2}}{d x} \cdot \sin ^{2} \phi \cdot x+ \\
& T_{2}\left(\sin ^{2} \phi+\sin 2 \phi \cdot x \cdot \frac{d \phi}{d x}\right)
\end{aligned}
$$




$$
\begin{aligned}
& \frac{d^{2} y}{d x^{2}}= \frac{d^{2} T_{1}}{d x^{2}} \cdot x+2 \frac{d T_{1}}{d x}+\frac{d^{2} T_{2}}{d x^{2}} \cdot \sin ^{2} \phi \cdot x+ \\
& \frac{d T_{2}}{d x}\left(2 \sin ^{2} \phi+\sin 2 \phi \cdot x\right)+ \\
& T_{2}\left[\frac{d^{2} \phi}{d x^{2}} \cdot \sin 2 \phi \cdot x+2\left(\frac{d \phi}{d x}\right)^{2} \cdot \cos 2 \phi \cdot x+\right. \\
&\left.2 \frac{d \phi}{d x} \cdot \sin 2 \phi\right] \\
& \frac{d^{3} y}{d x^{3}}= \frac{d^{3} T_{1}}{d x^{3}} x+3 \frac{d^{2} T_{1}}{d x^{2}}+\frac{d^{3} T_{2}}{d x^{3}} \cdot \sin ^{2} \phi \cdot x+ \\
& \frac{d^{2} T_{2}}{d x^{2}}\left(3 \sin { }^{2} \phi+\frac{d \phi}{d x} \cdot \sin 2 \phi \cdot x+\sin 2 \phi \cdot x\right)+ \\
& \frac{d T_{2}}{d x}\left[\frac{d^{2} \phi}{d x^{2}} \sin 2 \phi \cdot x+2\left(\frac{d \phi}{d x}\right)^{2} \cdot \cos 2 \phi \cdot x+\right. \\
&\left.2 \frac{d \phi}{d x}(2 \sin 2 \phi+\cos 2 \phi \cdot x)+\sin 2 \phi\right] \times \\
& T_{2}\left[\frac{d^{3} \phi}{d x^{3}} \sin 2 \phi \cdot x+3 \frac{d^{2} \phi}{d x^{2}} \sin 2 \phi+\right. \\
& 6 \frac{d^{2} \phi}{d x^{2}} \frac{d \phi}{d x} \cos 2 \phi \cdot x-4\left(\frac{d \phi}{d x}\right)^{3} \sin 2 \phi \cdot x+ \\
&\left.6\left(\frac{d \phi}{d x}\right)^{2} \cos 2 \phi\right]
\end{aligned}
$$

where

$$
\begin{aligned}
& T_{1}=\frac{\ln (G) \cdot\left(1-\sqrt{R_{1} R_{2}} G\right)^{2}}{\left(1-R_{1}\right)(G-1)\left(1+R_{2} G\right)} \\
& T_{2}=\frac{4 \ln (G) \cdot \sqrt{R_{1} R_{2}} G}{\left(1-R_{1}\right)(G-1)\left(1+R_{2} G\right)} \\
& \frac{d T_{1}}{d x}=\frac{d T_{1}}{d G} \cdot \frac{d G}{d x}, \frac{d T_{2}}{d x}=\frac{d T_{2}}{d G} \cdot \frac{d G}{d x} \\
& \frac{d G}{d x}=-\frac{\Gamma \cdot g_{0} \cdot L}{\exp \left[\left(\alpha-\frac{\Gamma \cdot g_{0}}{x+1}\right) L\right](x+1)^{2}} \\
& \frac{d \phi}{d x}=\frac{g_{0} L b}{2(x+1)^{2}} .
\end{aligned}
$$

The selection of the reference point $x_{0}$ for the Taylor series expansion is of importance as well. Here more attention should be paid to the fact that the bistability in SOA occurs at the long wavelength side of the cavity resonance wavelength, as pointed out by aforementioned literatures [3, 6]. Therefore, it can be inferred that in the region that bistability occurs the phase detuning factor $\phi$ is negative. This characterizes the interval of $x$ in which we want to examine the feature of the first order derivative of $y$. Of course, the reference point should be chosen such that the phase detuning factor is less than zero. In this paper, $x_{0}$ is taken as half of the point at which $\phi$ is equal to 0 . Using (4), it can be derived as

$$
x_{0}=\frac{-\phi_{0}}{\Gamma g_{0} L b+2 \phi_{0}} \text {. }
$$

Substitute (9)-(12) into (8), $y$ is re-expressed as a cubic function of $x$. Differentiating both sides of (8), we can get

$$
\frac{d y}{d x}=\frac{F_{3}}{2} x^{2}+\left(F_{2}-F_{3} \cdot x_{0}\right) x+\left(\frac{F_{3}}{2} \cdot x_{0}^{2}-F_{2} \cdot x_{0}+F_{1}\right) \text {. (13) }
$$

Let (13) equal to 0 , then a quadratic equation is achieved in the form

$$
\frac{F_{3}}{2} x^{2}+\left(F_{2}-F_{3} \cdot x_{0}\right) x+\left(\frac{F_{3}}{2} \cdot x_{0}^{2}-F_{2} \cdot x_{0}+F_{1}\right)=0 .
$$

which owns the discriminant $\Delta$ as

$$
\Delta=F_{2}^{2}-F_{1} \cdot F_{3} \text {. }
$$

Equation (14) would own two real roots when $\Delta$ is greater than 0 . Thus the necessary condition for bistability of SOAs can be described as

$$
\Delta=F_{2}^{2}-F_{1} \cdot F_{3}>0 .
$$

When this condition is satisfied, an IO hysteresis loop can be acquired. Next, based on the above results, we would derive the analytical expression of the hysteresis loop width.

When $\Delta>0$, the two real roots of (14) are

$$
\begin{aligned}
& x_{1}=\frac{F_{3} \cdot x_{0}-F_{2}+\sqrt{\Delta}}{F_{3}} \\
& x_{2}=\frac{F_{3} \cdot x_{0}-F_{2}-\sqrt{\Delta}}{F_{3}} .
\end{aligned}
$$

Substitute (17) and (18) into (7), the switch-up power $P_{\text {up }}$ and switch-down power $P_{\text {down }}$ of the input beam for the hysteresis loop in the SOA can be easily obtained:

$$
\begin{aligned}
P_{\text {up }}= & \frac{\ln \left(G_{2}\right) \cdot\left[\left(1-\sqrt{R_{1} R_{2}} G_{2}\right)^{2}+4 \sqrt{R_{1} R_{2}} G_{2} \sin ^{2} \phi_{2}\right]}{\left(1-R_{1}\right)\left(G_{2}-1\right)\left(1+R_{2} G_{2}\right)} . \\
& x_{2} \cdot I_{s} \cdot A_{\text {in }}
\end{aligned}
$$




$$
\begin{aligned}
& P_{\text {down }}= \frac{\ln \left(G_{1}\right) \cdot\left[\left(1-\sqrt{R_{1} R_{2}} G_{1}\right)^{2}+4 \sqrt{R_{1} R_{2}} G_{1} \sin ^{2} \phi_{1}\right]}{\left(1-R_{1}\right)\left(G_{1}-1\right)\left(1+R_{2} G_{1}\right)} . \\
& x_{1} \cdot I_{s} \cdot A_{\text {in }}
\end{aligned}
$$

where $G_{1}$ and $\phi_{1}$ denote the values of $G$ and $\phi$ at $x=x_{1}, G_{2}$ and $\phi_{2}$ denote the values of $G$ and $\phi$ at $x=x_{2}$, and $A_{\text {in }}$ represents the area of the input beam. Combining (19) and (20), we can derive the bistable loop width $H$ as

$$
H=P_{\text {up }}-P_{\text {down }} .
$$

\section{Numerical simulations}

In the numerical simulation, the parameter values are taken as follows, $A_{\text {in }}=0.8 \mu \mathrm{m}^{2}, L=500 \mu \mathrm{m}$, $R_{1}=R_{2}=0.3, \quad n_{0}=1.5 \times 10^{24} \mathrm{~m}^{-3}, \quad a=2.7 \times 10^{-20} \mathrm{~m}^{2}$, $\alpha=1000 \mathrm{~m}^{-1}, b=4.8$, and $\Gamma=0.1$. Figs. 1 and 2 plot the dependence of $\Delta$ on the initial phase factor and the normalized carrier concentration, respectively. According to the above derived criterion for the occurrence of hysteresis, when $\Delta$ is greater than 0 , the bistable loop could be found. In Fig. 1, it can be seen that $\Delta$ is positive when $\phi_{0}$ is in the interval $[-0.78 \pi,-0.18 \pi]$ as the normalized carrier concentration is 0.95 . Further numerical simulations are carried out under the same parameters, which show that in this interval the SOA would appear the bistable behaviors. Similarly, Fig. 2 reveals that if the normalized carrier density is greater than $0.88, \Delta$ is positive when the initial phase detuning factor $\phi_{0}$ is $-0.22 \pi$. Numerical calculations also exhibit that just in this region the bistable loop can be observed. All these confirm the effectiveness of the derived criterion (16) for the hysteresis in the SOAs.

Figure 3 displays the numerical and analytical results of the switch-up power as a function of the normalized carrier concentration for various initial phase detuning factors. Obviously, the discrepancy between the simulations and the derived formulae is negligible. Moreover, it can be seen that $P_{\text {up }}$ dwindles as the carrier density increases, and with the enhancement of the initial phase detuning, $P_{\text {up }}$ rises up drastically. In Fig. 4, the tendency of the switch-down power $P_{\text {down }}$ is plotted, as the normalized carrier concentration grows up for various initial phase detuning factors. The analytical results show a good agreement with the numerical ones. Furthermore, $P_{\text {down }}$ possesses a similar tendency to $P_{\text {up }}$, when the carrier concentration or the initial phase detuning factor increases. In Figs. 5 and 6 , the bistable loop width is shown as a function of the normalized carrier density and the initial phase detuning factors, respectively. It is observed that analytical calculations are slightly smaller than numerical simulations. Meanwhile, the hysteresis loop becomes wider with the increment of either the carrier density or the initial phase detuning factor.

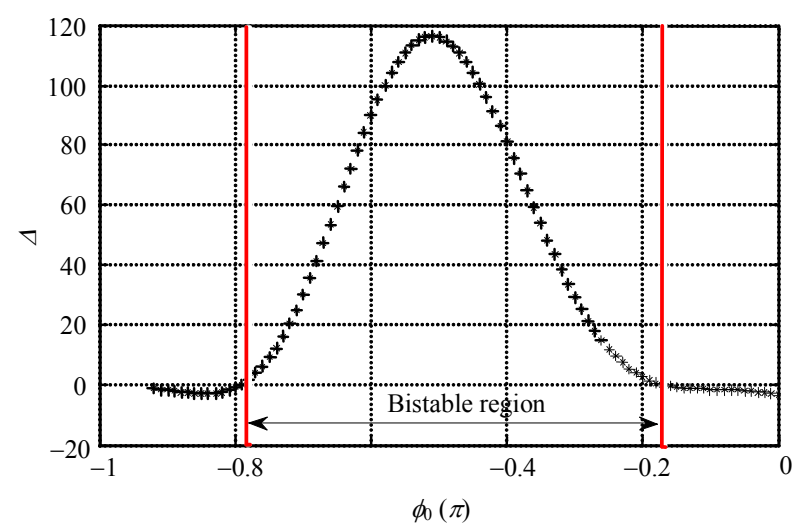

Fig. 1 Dependence of $\Delta$ on the initial phase detuning factor $\phi_{0}, n / n_{\mathrm{th}}=0.95$.

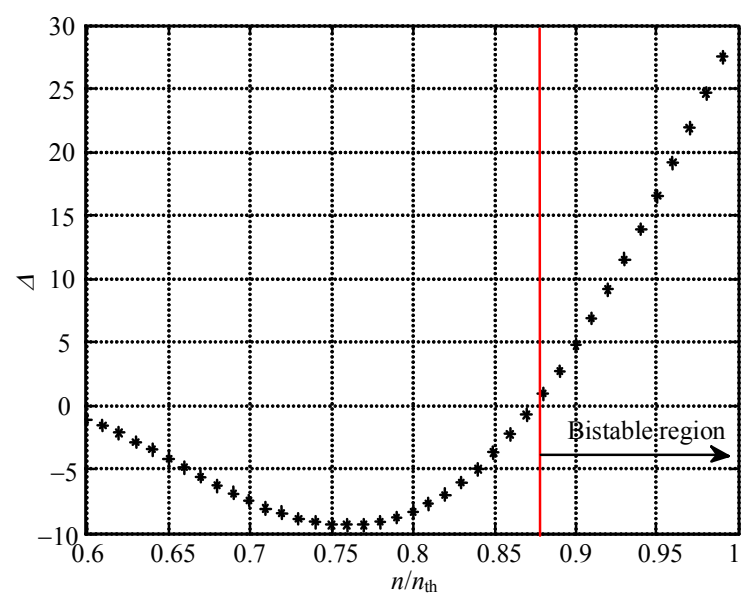

Fig. 2 Dependence of $\Delta$ on the normalized carrier concentration $n / n_{\mathrm{th}}, \phi_{0}=-0.22 \pi$. 




Fig. 3 Jump-up power $P_{\text {up }}$ versus normalized carrier density $n / n_{\text {th }}$ for three cases: (a) $\phi_{0}=-0.22 \pi$, (b) $\phi_{0}=-0.42 \pi$, and (c) $\phi_{0}=$ $-0.62 \pi$ (The solid lines and the symbols represent the numerical simulations and the analytical results, respectively).

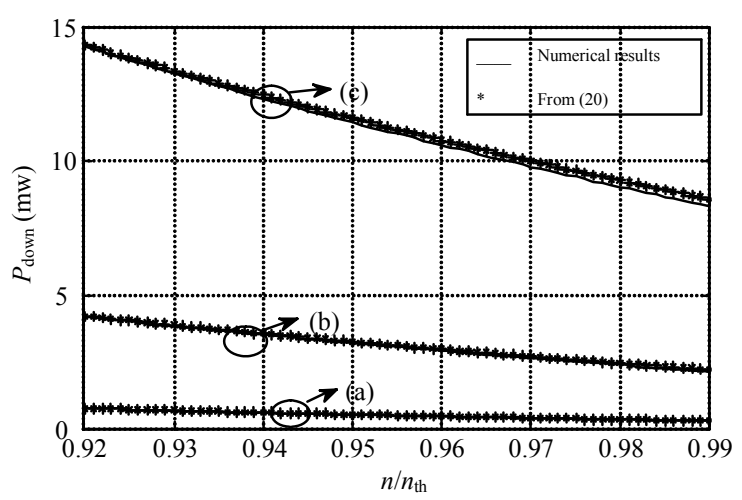

Fig. 4 Comparison between analytical and numerical results of the jump-down input power $P_{\text {down }}$ for three cases: (a) $\phi_{0}=$ $-0.22 \pi$, (b) $\phi_{0}=-0.42 \pi$, and (c) $\phi_{0}=-0.62 \pi$ (Other parameters are the same as Fig. 3).

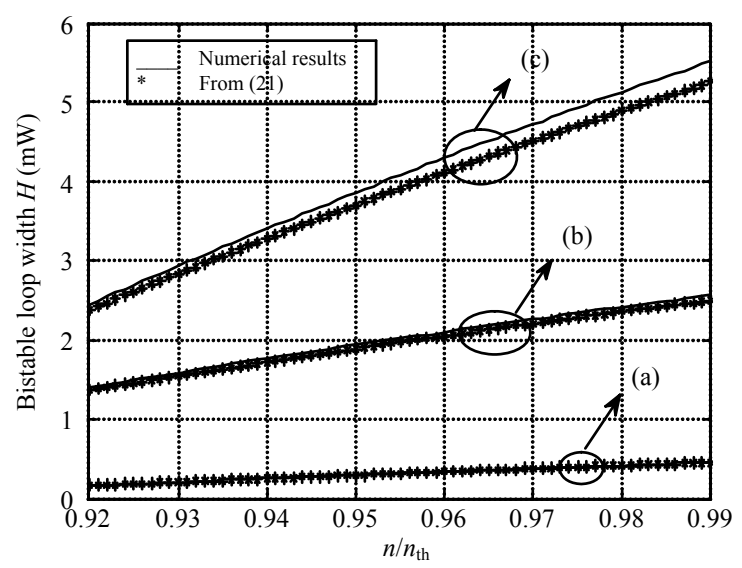

Fig. 5 Bistable loop width as a function of the normalized carrier density $n / n_{\text {th }}$ for three cases: (a) $\phi_{0}=-0.22 \pi$, (b) $\phi_{0}=$ $-0.42 \pi$, and (c) $\phi_{0}=-0.62 \pi$ (The solid lines and the symbols represent the numerical simulations and the analytical results, respectively).

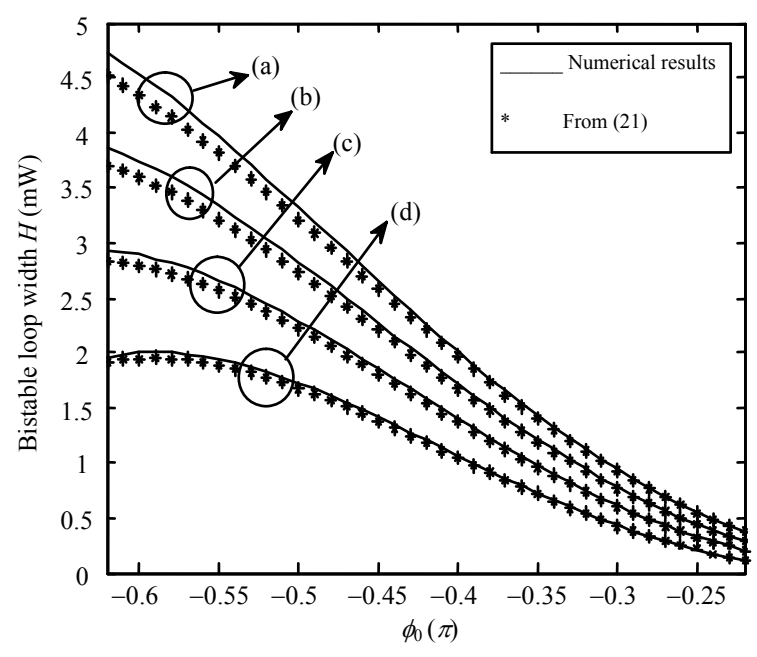

Fig. 6 Analytical and numerical results of bistable loop width as a function of the initial phase detuning factor $\phi_{0}$ for four cases: (a) $n / n_{\text {th }}=0.97$, (b) $n / n_{\text {th }}=0.95$, (c) $n / n_{\text {th }}=0.93$, and (d) $n / n_{\text {th }}=0.91$.

\section{Conclusions}

In summary, based on Adams's relationship between the $z$-independent intracavity intesnsity $I_{\text {av }}$ and the incident intensity $I_{\text {in }}$ in an SOA, we deduce the criterion for the hysteresis in SOAs by expanding $I_{\text {in }}$ as a Taylor series in terms of $I_{\mathrm{av}}$. As a result, the analytical expressions of the switch-up power, the switch-down power of the incident beam, and the width of the hysteresis loop in SOAs are derived for the first time. Numerical simulations are performed to test the effectiveness of the criterion and the accuracy of the expressions. The comparisons reveal that our analytical formulae are in good agreement with the numerical results. These conclusions would afford a valuable way for future investigations into the bistability in SOAs and other devices with a similar structure.

Open Access This article is distributed under the terms of the Creative Commons Attribution 4.0 International License (http://creativecommons.org/licenses/by/4.0/), which permits unrestricted use, distribution, and reproduction in any medium, provided you give appropriate credit to the original author(s) and the source, provide a link to the Creative Commons license, and indicate if changes were made. 


\section{References}

[1] P. M. Mejias and R. M. Herrero, "Bistable fiber-optics interferonetric sensor," Applied Optics, 1988, 27(5): $811-813$.

[2] S. H. Shehadeh, D. Steup, and J. Weinzierl, "Cascaded linear and nonlinear optical resonators: Towards a smart deflection sensor," in Microsystems and Nanoelectronics Research Conference, Canada, Oct. 13-14, 2009.

[3] P. Pakdeevanich and M. J. Adams, "Measurements and modeling of reflective bistability in $1.55-\mu \mathrm{m}$ laser diode amplifiers," IEEE Journal of Quantum Electronics, 1999, 35(12): 1894-1903.

[4] N. F. Mitchell, J. O'Gorman, and J. Hegarty, “Optical bistability in asymmetric Fabry-Perot laser-diode amplifiers," Optics Letters, 1994, 19(4): 269-271.
[5] P. Wen, Michael S, M. Gross, and S. Esener, "Observation of bistability in vertical-cavity semiconductor optical amplifier (VCSOA)," Optics Express, 2002, 10(22): 1273-1278.

[6] A. Hurtado, A. Gonzalez-Marcos, and J. A. Martin-Pereda, "Modeling reflective bistability in vertical-cavity semiconductor optical amplifiers," IEEE Journal of Quantum Electronics, 2005, 41(3): 376-383.

[7] C. F. Marki, D. R. Jorgesen, H. J. Zhang, P. Wen, and S. C. Esener, "Observation of counterclockwise, clockwise and butterfly bistability in $1550 \mathrm{~nm}$ VCSOAs," Optics Express, 2007, 15(8): 4953-4959.

[8] W. L. Zhang and S. F. Yu, "Bistabilities of birefringent vertical-cavity semiconductor optical amplifiers with antiresonant reflecting optical waveguide," IEEE Journal of Quantum Electronics, 2010, 46(1): 11-18. 